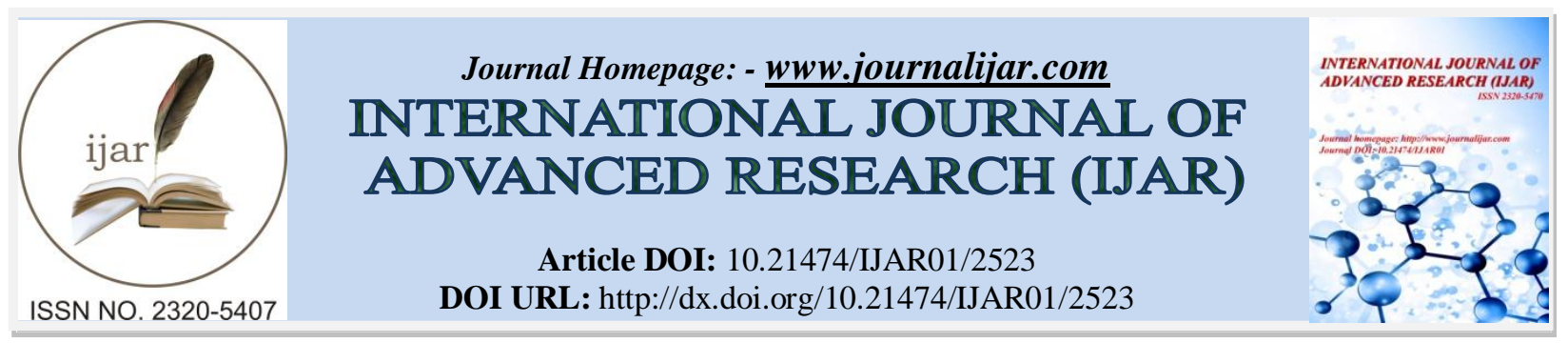

RESEARCH ARTICLE

\title{
THE EFFECT OF COMPUTER GAMES ON THE DEVELOPMENT OF ANXIETY DISORDERS IN STUDENTS.
}

\begin{abstract}
*Mezianaya K., Karaneuski K., Dick S., Davidovski A. and Yashin K.
The Belarusian State University of Computer Science and Radio Electronics, 6 ul. Brovki, Minsk 220027 Republic of Belarus.
\end{abstract}

\section{Manuscript Info}

anc...................

Manuscript History

Received: 25 October 2016

Final Accepted: 23 November 2016

Published: December 2016

\section{Key words:-}

Addiction (psychology), computers games, gambling, insomnia, anxiety disorders.

\section{Abstract}

Introduction of information and computer technologies in the sphere of entertainment has resulted in negative influence on the health of users. The problem of the impact of virtual space on a person is being studied in many countries. This article presents the results of studying the problem of computer addiction and its effects on sleep disorders and development of anxiety disorders in the computer media users.

The study involved 65 students, among them males 44 (67.7\%), studying technical specialties in one of the universities in Minsk city. The analysis of computer addiction was carried out by means of the questionnaire developed by Mezianaya K. N., Karaneuski K. M., and Yashin, K. D., titled "Method of Screening Diagnostics of Computer Addiction and Its Health Effects" [The Official Journal of the Republic of Belarus, 2015]. In order to detect anxiety disorders we used the anxiety self-assessment scale by Spielberger-Hanin.

It was found that $21.5 \%$ of the students surveyed are subject to the computer addiction. 36 persons $(55.4 \%)$ of the total number surveyed suffer from intrasomnia; they used to say to themselves in a dream: "I just dream it!" Waking up due to fear, anxiety and unpleasant dreams were revealed in 49 persons $(76.4 \%)$. Analysis of anxiety disorders in students showed that 27 persons $(41.5 \%)$ had a high level of trait anxiety and 38 persons $(58.5 \%)$ got a medium level.

The study found that computer addiction is one of the causes of sleep and anxiety disorders in students.

Copy Right, IJAR, 2016,. All rights reserved.

\section{Introduction:-}

Anxiety is one of the individual psychological attributes of a personality. It is closely related to mental adaptation mechanisms. The increased level of anxiety in patients with chronic mental tension is a factor that affects individual adaptation. It promotes the formation of mental and psychosomatic diseases. According to clinical and epidemiological studies more than $9 \%$ of the world population suffer from any anxiety disorder, with average age of its onset being 25 to 27 years. Studies by Breitholz and Johansson confirmed the cognitive theory of anxiety disorder development and performed the analysis of cognitive structures of anxiety spectrum ("anxious thinking") [Breitholtz E., 1999].

The clinical presentation of anxiety disorders is dominated by irrational conclusions, persistent inexplicable anxiety, and a variety of somatic symptoms, which leads to neurosis and social and labour maladjustment. One of the most 
frequent manifestations of anxiety is night sleep disorders [Strygin K., 2011]. Epidemiological studies have shown that in case of anxiety disorders sleep disorders are detected in 44 to $81 \%$ : in $68 \%$ of cases they occur simultaneously with the development of anxiety [Ford D., 1989], and in 15\% appear shortly after onset of anxiety disorders [Abad V., 2005]. These disorders are often leading ones, and even the only manifestations of anxiety. However, the causes of sleep and anxiety disorders in the virtual world users, and in particular video gamers and movie watchers, have not yet been well studied so far [Davidovsky A., 2015; Mezianaya K., 2015; Kaess M., 2014].

\section{Study objective:-}

To study the students with anxiety disorders using information and computer technology (ICT) for the purpose of entertainment.

\section{Study tasks:-}

1. To study the prevalence of computer addiction.

2. To study the level of anxiety disorders among students and to analyse their relationship with sleep disorders.

3. To investigate the effect of virtual world type on the development of insomnia as one of the symptoms of anxiety disorders.

\section{Materials and Methods:-}

The study involved 65 information technology students from one of the technical universities of Minsk at the age of 18 and older, among them males $44(67.7 \%)$ and females 21 (32.3\%). All students gave the informed consent. An anonymous continuous survey was conducted in the second semester of the academic year (March 2016). The average age of the respondents was $19.7( \pm 1.5)$ years. The average duration of computer use (games and Internet) was 9 years. All students at the time of the study had personal computers and were using Internet.

To conduct the study, Mezianaya K.N., Yashin K.D., and Karaneuski K.M. have developed the questionnaire titled "Method of Screening Diagnostics of Computer Addiction and Its Effect on Physical and Mental Health" (Application for an Invention "Method for Diagnosing the Human Mental State (Options)" \# a 20140678 dated 08.30.2015, the authors: Mezianaya K.N., Yashin K.D., Karaneuski K.M.) [The Official Journal of the Republic Belarus, 2015]. The questionnaire includes questions on the time spent in the virtual world, the structure of sleep and the nature of dreams. Six types of virtual world use were analyzed: computer games, social networking, surfing the Internet, watching movies, including TV series and video clips, cybersex, exchange trading and gambling, according to the classification adopted in the CIS countries [Egorov A., 2007]. Students were allowed to mark more than one answer. Evaluation of the results was carried out by a three-point scale depending on the number of virtual world types: $1-2$ types ( 1 point), $3-4$ types ( 2 points), $5-6$ types ( 3 points). For the analysis of time devoted to virtual world entertainment a time period of 7 days (168 hours) was applied, which was divided into three intervals: 1 to 24 hours, 25 to 39 hours, 40 hours or more per week. The intervals were assessed by a three-point scale: the minimum score was 1 for the minimal amount of time. We also analyzed the number of hours spent at the computer per day by the same three intervals with a similar assessment. To analyze behavioural disorders and the symptoms characteristic of this type of addiction, we revealed obsession, neglect and control disorder [Koronczai B., 2011]. The responses to each of the three questions of the questionnaire were evaluated by a three-point scale: Never -1 point, Sometimes -2 points, Often -3 points. The result is the total number of points.

At the same time the survey was conducted among students on the anxiety self-assessment scale developed by Spielberger-Hanin [Spielberger C., 1983].

\section{Statistics:-}

Statistical analysis of the data was carried out by contingency tables with calculation of $\chi^{2}$ (Chi-square or Pearson criterion). To establish the correlation measure between the symptoms coefficient $\varphi$ was used, where 0 was loose correlation, and 1 was strong. $\chi^{2}$ (Pearson criterion) was calculated (at the confidence level of $\alpha=0.05$ ) for four symptoms: computer addiction, intrasomnia, high level of trait anxiety and the combine using computer games with watching movies. Differences were considered statistically significant at $\mathrm{p}<0.05$ [Glance S.,1998].

The results were processed using Microsoft Office Excel 2010 and STATISTICA 10.0 package. 


\section{Results:-}

As shown by the analysis of the survey results, the majority of students, 48 persons (73.8\%) were using three or more types of the virtual world for the purpose of entertainment, which is time-consuming: 46 persons (70.8\%) spent at the computer 25 or more hours a week. Among those surveyed 44 persons were using computer games $(67.7 \%)$, while 30 of them (46.2\%) combined gaming activity with watching movies and television series. Analysis of pathopsychological characteristics showed that according to Spielberger- Hanin scale all subjects had increased levels of trait anxiety: high level was revealed in 27 persons (41.5\%), and medium level in 38 persons (58.5\%).

The results of the survey conducted by the questionnaire, which we had developed showed that 14 persons (21.5\%) suffered from computer addiction, 34 persons $(52.3 \%)$ were at risk of its progression. Among computer-addicted persons insomnia was found in 13 subjects, and many of them were prone to anxiety during the night.

It was established that 36 persons (55.4\%) of the total number of surveyed students suffered from intrasomnia: they used to say to themselves in a dream: "I just dream it!", which was a symptom of superficial sleep and anxiety [Freud S., 2013]. Among them, 19 persons had high levels of trait anxiety and 8 persons were seeing dreams about dismembered human bodies. Table 1 shows the results of data analysis by contingency tables with calculation of Pearson criterion $\chi 2$ and coefficient $\varphi$ to determine the statistical relationship between computer addiction, high level of trait anxiety and intrasomnia. It was found that the relationship between these symptoms is statistically significant at the level of $\mathrm{p}<0.05$ (Table 1 ).

Table 1:- Relation of high level of trait anxiety (by Spielberger-Hanin scale) to computer addiction and intrasomnia, as determined by the diagnostic questionnaire aimed to reveal the computer addiction and the consequences of its effects on health.

\begin{tabular}{|l|c|c|c|}
\hline \multirow{2}{*}{} & \multicolumn{3}{|c|}{ High level of trait anxiety } \\
\cline { 2 - 4 } & Pearson Chi-square & Phi for 2 x 2 tables & $\mathrm{p}$ value \\
\hline $\begin{array}{l}\text { Intrasomnia } \\
\text { "I just dream it" }\end{array}$ & 5.540 & 0.292 & 0.018 \\
\hline Computer addiction & 5.778 & 0.298 & 0.016 \\
\hline
\end{tabular}

These results suggest that individuals with computer addiction have higher levels of anxiety disorder than the rest of the population. As is known, in case of an anxiety disorder there is a tendency to irrational judgment and a sense of impending danger. The survey showed that 16 students $(24.6 \%)$ of the respondents did not feel safe in everyday life (Spielberger-Hanin's scale, Question 33).

Analysis of the impact of certain virtual world types on the psycho-emotional sphere of users revealed that computer games and movies and TV series exert a significant influence. A statistical relation between the following symptoms was established: combination of computer games with watching movies and TV series and intrasomnia ("I just dream it!") (Table 2). This indicates that the combination of games with movies increases the likelihood of sleep disorders, which contributes to the development of anxiety disorders in users.

Table 2:- Interrelation of symptoms: combining computer games with watching movies and intrasomnia.

\begin{tabular}{|c|c|c|c|}
\hline \multirow{2}{*}{$\begin{array}{c}\text { Intrasomnia "I just dream } \\
\text { it" }\end{array}$} & Pearson Chi-square & Phi for 2 x 2 tables & p value \\
\cline { 2 - 4 } & 30.121 & 0.681 & 0.001 \\
\hline
\end{tabular}

The study found that 49 persons (76.4\%) used to wake up from fear, anxiety, and/or unpleasant dreams. The cause of these disorders can be nightmares during sleep [World Health Organization, 1989]. The analysis has shown that the formation of students' anxiety during sleep was caused by the dream scenes: 23 persons (35.4\%) saw dead human bodies, while 9 persons saw bloodied and dismembered bodies. A high or medium level of trait anxiety was revealed in all of them. This may indicate that the experiences induced by the virtual world (images, emotions, and actions) contribute to the development of chronic mental strain with insomnia, which leads to the formation of anxiety disorders. 


\section{Conclusion:-}

It was established during the study that anxiety disorders combined with insomnia have been formed in $41.5 \%$ of students using information and computer technology for entertainment. Insomnia was revealed in the computer addicted individuals in $92.9 \%$ of cases, with the anxiety attack during the night experienced by $71.4 \%$. In the structure of sleep disorders in students who combine computer games with watching movies and TV series, the most frequent one is intrasomnia. We have found that the occurrence of negative dreams (when they tell themselves in a dream, "I just dream it") may be an indicator of abnormality in physiological mechanisms of organization and sustaining of sleep in individuals with high levels of anxiety. Further studies are needed using neurophysiologic diagnostic methods, including polysomnographic examination, which will determine the nature of the effect of different virtual world types and computer games genres on the mental health of users.

\section{References:-}

1. The Official Journal of the Republic of Belarus. 2015. № 4. C.6. URL: http: // belgospatent / org, by / database / index.php pref $=$ inv \& $\operatorname{lng}=$ ru \& pa?

2. Breitholtz E., Johansson B., Ost L.G (1999). Cognitions in generalized anxiety disorder and panic disorder patients. A prospective approach. Behavior Research Therapy. 37(6):533-44.

3. Strygin K.M., Yumatov E.A,.Levin Ya.I. (2011). Interrelation of personal features and characteristics of human nocturnal sleep // Topical Somnology Problems. - Moscow. - 63.

4. Ford D.E., Kamerow D.B. Epidemiologic study of sleep disturbances and psychiatric disorders // JAMA. 1989. - Vol. 262. - P. 1479-1484.

5. Abad V.C., Guilleminaut C. 2005 Sleep and Psychiatry. Dialogues in Clinical Neuroscience. 7(4): 291-303.

6. Davidovsky A.G., Mezianaya K. N., Yashin K. D., Karaneuski K.M.// Influence of computer games on the sleep quality and the nature of dreams in students. // European science review. - 2015. - №. 5 - 6.S. 130-133.

7. Mezianaya, K. N., Yashin, K. D., Karaneuski, K. M., Dick C. K., Davidovski A. G., Pishchova A. V., Yashina T. K. // Analysis of sleep disorders and emotional disturbances in the students engaged in computer games // Juorn. Medical News. 2015. № 10. P.24-26.

8. Kaess M., Durkee T., Brunner R. Pathological Internet use among European adolescents psychopathology and self-destructive behaviours.// Journ. Eur. Child Adolesc Psychiatry. 2014. V.23. P.1093-1102.

9. Egorov A. Ju. Non-chemical Dependencies. St. Petersburg: Rech, 2007.- 190 p.

10. Koronczai B., Urbán R., Kökönyei, Paksi B., Kun B., Arnold A. Confirmation of the Three-Factor Model of Problematic Internet Use on Off-Line Adolescent and Adult Samples.// Journ. Cyberpsychol Behav Soc. Netw. 2011. V.14. P. 657-664.

11. Spielberger C. D. State-Trait Anxiety Inventory. Consulting Psychologists Press; Palo Alto (CA): 1983.

12. Glance S.A. Primer of Biostatistics. Medical Statistics. Practica., Moscow, 1998 p. 456.

13. Freud S. "The Interpretation of Dreams." [Electronic resource] URL: http://poetry.rapgenius.com/albums/Sigmund-freud/The-interpretation-of-dreams. (visit date 01.07.2016).

14. World Health Organization (1989) International Classification of Diseases (ICD-10). Geneva: WHO.

15. The authors express gratitude to Ms Stepanova M.D., Associate Professor , Chair of Intelligent Information Technologies, the Belarusian State University of Informatics and Radioelectronics, for her support and advice in preparation of this article. 